\title{
The first law of black hole mechanics in the Einstein-Maxwell theory revisited
}

\author{
Zachary Elgood, ${ }^{a}$ Patrick Meessen ${ }^{b, c}$ and Tomás Ortín ${ }^{a}$ \\ ${ }^{a}$ Instituto de Física Teórica UAM/CSIC, \\ C/ Nicolás Cabrera, 13-15, C.U. Cantoblanco, E-28049 Madrid, Spain \\ ${ }^{b}$ HEP Theory Group, Departamento de Fisica, Universidad de Oviedo, \\ Avda. Calvo Sotelo s/n, E-33007 Oviedo, Spain \\ ${ }^{c}$ Instituto Universitario de Ciencias y Tecnologías Espaciales de Asturias (ICTEA), \\ Calle de la Independencia, 13, E-33004 Oviedo, Spain \\ E-mail: zachary.elgood@uam.es, meessenpatrick@uniovi.es, \\ tomas.ortin@csic.es
}

ABSTRACT: We re-derive the first law of black hole mechanics in the context of the EinsteinMaxwell theory in a gauge-invariant way introducing "momentum maps" associated to field strengths and the vectors that generate their symmetries. These objects play the role of generalized thermodynamical potentials in the first law and satisfy generalized zeroth laws, as first observed in the context of principal gauge bundles by Prabhu, but they can be generalized to more complex situations. We test our ideas on the $d$-dimensional Reissner-Nordström-Tangherlini black hole.

Keywords: Black Holes, Classical Theories of Gravity

ARXIV EPRINT: 2006.02792 


\section{Contents}

1 Introduction 1

2 Covariant Lie derivatives and momentum maps 4

2.1 Lie-Maxwell derivatives 4

2.2 Lie-Lorentz derivatives $\quad 6$

3 The Einstein-Maxwell action and the RNT solutions 10

$\begin{array}{ll}3.1 \text { Action and equations of motion } & 10\end{array}$

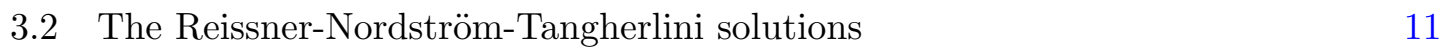

4 Wald-Noether charge for the E-M theory 13

5 The first law of black hole mechanics in the E-M theory 15

$\begin{array}{lll}6 & \text { Discussion } & 17\end{array}$

\section{Introduction}

Black-hole thermodynamics ${ }^{1}$ is probably one of the most active fields of research in Theoretical Physics. It interconnects seemingly disparate areas of Physics such as Gravity, Quantum Field Theory and Information Theory providing deep insights in all of them.

Black-hole thermodynamics originates in the analogy between the behaviour of the area of the event horizon $A$ and the second law obeyed by the thermodynamic entropy $S$ noticed by Bekenstein [2,3] in the results obtained by Christodoulou and Hawking [4-7]. Shortly afterwards, in ref. [8] Bardeen, Carter and Hawking extended this analogy by proving another three laws of black hole mechanics similar to the other three laws of thermodynamics involving the event horizon's surface gravity $\kappa$ and angular velocity $\Omega$ and the black hole's mass $M$. However, the analogy was only taken seriously after Hawking's discovery that black holes radiate as black bodies with a temperature $T=\kappa / 2 \pi[9]$, which implied the relation $S=A / 4$, both in $c=G_{N}=\hbar=k=1$ units.

Ever since the formulation of these four laws, it has been tried to extend their domain of application and validity with the inclusion of matter fields and terms of higher-order in the curvature, for instance. In refs. [10-12] Wald and collaborators developed a new approach to demonstrate the first law of black hole mechanics in general diffeomorphism-invariant theories, beyond General Relativity. Since the surface gravity relation to the Hawking temperature only depends on generic properties of the event horizon, the quantity whose variation it multiplies in the first law is naturally associated to the Bekenstein-Hawking

\footnotetext{
${ }^{1}$ For a recent review on black-hole thermodynamics with many references see, e.g. [1].
} 
entropy $S$. This quantity, often called Wald entropy, is just $A / 4$ in General Relativity but, in more general theories, there can be additional terms which can be understood, for instance, as $\alpha^{\prime}$ corrections in Superstring Theories [13-18].

In the presence of matter fields, Wald's proof of the first law of black-hole mechanics had to be re-examined because one of the main assumptions refs. [11, 12] is that all matter fields behave as tensors and, simply put, there are no tensor fields apart form the metric and scalar fields (if any); all of them have some sort of gauge freedom and their transformations under diffeomorphisms are always coupled to gauge transformations. Indeed, as is wellknown, fermionic fields coupled to gravity transform under a local Lorentz group as spinors and bosonic fields must transform under some gauge group if unwanted, typically negativeenergy, states are to be eliminated. The only scalar in the Standard Model, the Higgs field, is, in fact, a $\mathrm{SU}(2)$ doublet.

The simplest matter field that, coupled to gravity, allows for black-hole solutions is the Maxwell field $[19,20]$. The presence of the field introduces an additional term of the form $\Phi d Q$ in the first law which takes into account the changes in the mass of the black hole when its charge $Q$ changes. In this term $\Phi$ is the electric potential on the horizon and a generalized zeroth law states that it takes a constant value over the horizon. The value of $\Phi$ is customarily taken to be $k^{\mu} A_{\mu}$, where $k^{\mu}$ is the Killing vector for which the event horizon is its associated Killing horizon and where it is assumed that the electromagnetic field is in a gauge in which $\Phi$ is, indeed, constant.

This definition of $\Phi$ is clearly not gauge-invariant. This is a problem of principle, ${ }^{2}$ which, as we are going to show, is related to the more fundamental problem we were discussing: the fact that Wald's proof of the first law does not deal properly with fields which have some kind of gauge freedom. In Wald's proof, one considers diffeomorphisms which are symmetries of all the dynamical fields, but the naive definition of invariance of fields with gauge freedom under diffeomorphsisms through the standard Lie derivative is not gauge invariant. This problem affects the gravitational field itself when it is described in terms of the Vielbein instead of the metric.

A solution for this particular case was provided in ref. [22] by defining the variation of the Vielbein under diffeomorphisms through the Lie-Lorentz derivative refs. [23-27] which can be understood as a generalization of the Lie derivative which transforms covariantly under local Lorentz transformations. If the Vielbein is annihilated by the Lie-Lorentz derivative with respect to some vector field in some gauge it will be annihilated in any gauge and, as a matter of fact, the vector field will be a Killing vector field of the metric. The Lie-Lorentz derivative can be defined on all fields with Lorentz (spinor or vector) indices, a fact that has been used to extend the proof of the first law of black hole mechanics to supergravity in ref. [28].

A more general mathematically rigorous approach was proposed in $[29]^{3}$ using the formalism of principal gauge bundles which encompasses Yang-Mills and Lorentz fields

\footnotetext{
${ }^{2}$ There are other problems as well: in Wald's approach, the Noether charge, which contains a term in which $\Phi$ occurs, is evaluated over the bifurcation surface, but the Maxwell field of the Reissner-Nordström black hole turns out to be singular there in the traditional gauge [21].

${ }^{3}$ See also ref. [30] for a different take on this problem.
} 
but, unfortunately, not the Kalb-Ramond field or higher-rank form fields of string theory. ${ }^{4}$ Perhaps the most interesting result in that paper is the realization that all the zerothlaws (the constancy of the surface gravity, electric potential etc.), on the horizon fit into a common pattern. In this paper we are going to recover and reformulate this result in terms of the momentum map, using gauge-covariant derivatives in which this object plays a crucial rôle. ${ }^{5}$

Although gauge-covariant Lie derivatives are, perhaps, not the most mathematically rigorous tool one can use, they can be generalized to frameworks other than principal gauge bundles. ${ }^{6}$ Our goal in this paper is to show they can be consistently used in a simpler context (the Einstein-Maxwell theory described in terms of Vielbeins) and the objects to which the generalized zeroth law applies (here the surface temperature and the electric potential) are the gauge-invariant momentum maps associated to each gauge symmetry (Lorentz and $\mathrm{U}(1)$ ) evaluated over the horizon.

The emergence of the momentum map in this context may seem a bit strange; for instance, there is no mention of it in ref. [22] in spite of their use of the (gauge-covariant) Lie-Lorentz derivative. However, as we will show, the momentum map is indeed present in the Lie-Lorentz derivative and plays the same role of the momentum map we will introduce for the Maxwell case. As a matter of fact, gauge-covariant derivatives and the momentum map arise most naturally in the study of superalgebras of symmetry, when all the dynamical fields of a supergravity theory are left invariant by a set of supersymmetry and bosonic transformations that combine diffeomorphisms, gauge, local-Lorentz and local-supersymmetry transformations [35-38]. This object also plays a very interesting geometrical rôle in symmetric Riemannian spaces and in certain spaces of special holonomy when they admit Killing vectors that preserve their geometrical structures. When one wants to gauge the corresponding symmetries in theories with $\sigma$-models of that kind (typically supergravity theories) the momentum map plays an essential role in the definition of the gauge-covariant derivative [39].

This paper is organized as follows: in section 2 we introduce the gauge-covariant derivatives that we are going to use: Lie-Maxwell in section 2.1 and Lie-Lorentz in section 2.2. We also discuss the zeroth laws the respective momentum maps obey. This last section is essentially a review of the literature on the subject where we re-derive the formulae we are going to use in the main body of the paper using our conventions (those of ref. [38]). In section 3 we describe the Einstein-Maxwell theory in $d$ dimensions (action and equations of motion) in differential-form language and the $d$-dimensional Reissner-Nordström-Tangherlini black hole solutions. In section 4 we compute the Wald-Noether charge for this theory using the

\footnotetext{
${ }^{4}$ The first law has been proved for theories including one scalar and one $p$-form field in [31], although the gauge-invariance problem has not been discussed in it.

${ }^{5}$ In refs. [32, 33], which covers some of the topics studied here this object emerges as an "improved gauge transformation".

${ }^{6}$ In this paper we will not consider those more complicated cases involving higher-rank $p$-form fields with Chern-Simons terms which typically arise in Superstring/Supergravity theories. We will consider the case of the Kalb-Ramond field with Yang-Mills and Lorentz Chern-Simons terms in its field strength in ref. [34], where we will show how the gauge-covariant derivative approach with momentum maps that we introduce here provides a gauge-covariant, unambiguous results for the Wald-Noether charge.
} 
transformations based on the gauge-covariant Lie derivatives defined in section 2. Then, in section 5 we prove the first law for this system, identifying the Wald entropy, which we compute for the Reissner-Nordström-Tangherlini black hole solutions. In section 6 we briefly discuss our results and future directions of research.

\section{Covariant Lie derivatives and momentum maps}

One of the main ingredients in the proofs of the first law of black hole mechanics using Wald's formalism $[11,12]$ is the use of infinitesimal diffeomorphisms that leave invariant all the dynamical fields.

If we use the metric $g_{\mu \nu}$ as dynamical field, since the metric is just a tensor, its transformation under infinitesimal diffeomorphisms $\delta_{\xi} x^{\mu}=\xi^{\mu}(x)$ is given by (minus) the standard Lie derivative

$$
\delta_{\xi} g_{\mu \nu}=-£_{\xi} g_{\mu \nu}=-2 \nabla_{(\mu} \xi_{\nu)},
$$

which vanishes when $\xi^{\mu}$ is a Killing vector of $g_{\mu \nu}$. We will distinguish Killing vectors from generic vectors $\xi^{\mu}$ denoting them by $k^{\mu}$.

If, as we want to do here, we use as dynamical field the Vielbein $e^{a}{ }_{\mu}$ instead of $g_{\mu \nu}$, in order to define its symmetries, we face the well-known problem of the gauge freedom of $e^{a}{ }_{\mu}$, which in this context has been treated in refs. [22, 29]. The same happens with the electromagnetic potential $A_{\mu}$, which also has been treated in this context in ref. [29].

One way to deal with this problem is to define a gauge-covariant notion of Lie derivative. The Lie derivative in the corresponding principal bundle, used in ref. [29] provides the most rigorous definition of such a derivative. Here we will introduce a less sophisticated version that makes use of the so-called momentum map and which can be defined for more general fields such as the Kalb-Ramond 2-form of the Heterotic Superstring, which cannot be described in the framework of a principal bundle [34]. Gauge-covariant derivatives arise naturally in the commutator of two local supersymmetry transformations and in the construction of Lie superalgebras of supersymmetric backgrounds [35-38].

Due to its simplicity, we start with the Maxwell field.

\section{$2.1 \quad$ Lie-Maxwell derivatives}

The electromagnetic field $A_{\mu}$ is a field with gauge freedom: we must consider physically equivalent two configurations that are related by the gauge transformation

$$
\delta_{\chi} A_{\mu}=\partial_{\mu} \chi
$$

and, furthermore, as a general rule, it is not possible to give a globally regular expression of the electromagnetic field in a single gauge. ${ }^{7}$ However, the standard Lie derivative does not commute with these gauge transformations and gives different results in different gauges. This is why a gauge-covariant notion of Lie derivative is needed in this case.

In the subsequent discussion it is convenient to use differential-form language. In terms of the electromagnetic 1 -form potential $A \equiv A_{\mu} d x^{\mu}$, we define the electromagnetic

\footnotetext{
${ }^{7}$ The main example of this situation is the magnetic monopole [40].
} 
field strength 2 -form by $F=d A$ so that it satisfies the Bianchi identity $d F=0$. In components we have

$$
F \equiv \frac{1}{2} F_{\mu \nu} d x^{\mu} \wedge d x^{\nu}, \quad F_{\mu \nu}=2 \partial_{[\mu} A_{\nu]} .
$$

The field strength is invariant under the gauge transformations $\delta_{\chi} A=d \chi$ and we can treat it as a standard 2-form whose transformation under infinitesimal diffeomorphisms generated by $\xi^{\mu}$ is given by (minus) the standard Lie derivative which, on $p$-forms, acts as $£_{\xi}=\imath_{\xi} d+d \imath_{\xi} \cdot{ }^{8}$

Using the Bianchi identity we find that

$$
\delta_{\xi} F=-d \imath_{\xi} F
$$

If $\xi$ is a symmetry of all the dynamical fields, in which case we will denote it by $k$, we have that $\delta_{k} F=0$ and the above equation implies that, locally, there is a gauge-invariant function $P_{k}$ called momentum map such that ${ }^{9}$

$$
\imath_{k} F=-d P_{k} .
$$

$P_{k}$ is defined by this equation up to an additive constant that we will discuss later.

Let us now consider the variation of $A$ under infinitesimal diffeomorphisms, which, according to general arguments (see e.g. refs. [29, 38]) has to be given locally by a combination of (minus) the Lie derivative and a "compensating" gauge transformation generated by a $\xi$-dependent parameter $\chi_{\xi}$ which is to be determined by demanding that $\delta_{k} A=0$ when $\delta_{k} F=0$ :

$$
\delta_{\xi} A=-£_{\xi} A+d \chi_{\xi}=-\imath_{\xi} F+d\left(\chi_{\xi}-\imath_{\xi} A\right) .
$$

Then, taking into account eq. (2.5), we conclude that

$$
\chi_{\xi}=\imath_{\xi} A-P_{\xi},
$$

where $P_{\xi}$ is a function of $\xi$ which satisfies eq. (2.5) when $\xi=k$ and generates a symmetry of all the dynamical fields.

It is natural to identify the above transformation $\delta_{\xi} A$ with (minus) a gauge-covariant Lie derivative of $A$ that we can call Lie-Maxwell derivative

$$
\delta_{\xi} A=-\mathbb{L}_{\xi} A, \quad \quad \mathbb{L}_{\xi} A \equiv \imath_{\xi} F+d P_{\xi} .
$$

While this derivative does not enjoy the most important property of Lie derivatives $\left[£_{\xi}, £_{\eta}\right]=£_{[\xi, \eta]}$ for generic vector fields $\xi, \eta$, it is clear that it does for those that generate symmetries of $A$ and $F$ and annihilates them. This is certainly enough for us.

For stationary asymptotically-flat black holes, when the Killing vector $k$ is the one normal to the event horizon, the momentum map can be understood as the electric potential

\footnotetext{
${ }^{8}$ In our conventions, for a $p$-form $\omega^{(p)}$ with components $\omega^{(p)}{ }_{\mu_{1} \cdots \mu_{p}}, \imath_{\xi} \omega^{(p)}$ is the $(p-1)$-form with components $\left(\imath_{\xi} \omega^{(p)}\right)_{\mu_{1} \cdot \mu_{p-1}}=\xi^{\nu} \omega^{(p)}{ }_{\nu \mu_{1} \cdot \mu_{p-1}}$.

${ }^{9}$ The sign of $P_{k}$ is purely conventional.
} 
$\Phi$ which, evaluated on the horizon $\Phi_{\mathcal{H}}$, appears in the first law. ${ }^{10}$ In the early literature (see e.g. section 6.3 .5 of ref. [42]) it was assumed from the start that there is a gauge in which

$$
£_{k} A=\imath_{k} d A+d\left(\iota_{k} A\right)=0 .
$$

Then, the electric potential $\Phi$ was identified with $\imath_{k} A$ because, according to the above equation, $d \Phi=-\imath_{k} F$, which can be defined as the electric field for an observer associated to the time direction defined by $k$.

It is clear that $P_{k}$ can be identified with $\Phi$ (both satisfy the same equation). However, in a general gauge, it will not be given by just $\imath_{k} A$ and we will have to compute it. Nevertheless, the main property of $\Phi$, namely the fact that it is constant over the horizon (sometimes called generalized zeroth law) still holds because it is, actually, a property of $-\imath_{k} F$ based on the properties of $k$, the Einstein equations and the assumption that the energy-momentum tensor of the electromagnetic field satisfies the dominant energy condition.

\subsection{Lie-Lorentz derivatives}

The original motivation for the definition of a derivative covariant under local Lorentz transformations, often called the Lie-Lorentz derivative, was its need for the proper treatment of spinorial fields in curved spaces in such a way that the flat-space results were correctly recovered.

In Minkowski spacetime, fermionic fields transform in spinorial representations of the Lorentz group, which leaves invariant the spacetime metric $\left(\eta_{a b}\right)=\operatorname{diag}(+-\cdots-)$. Since generic spacetime metrics $g_{\mu \nu}$ do not have any isometries, the Lorentz group will not be realized as a group of general coordinate transformations (g.c.t.s) leaving invariant the spacetime metric. Weyl realized that, if one introduces an orthonormal base in cotangent space at a given point in spacetime

$$
\left\{e^{a}=e^{a}{ }_{\mu} d x^{\mu}\right\}, \quad e^{a}{ }_{\mu} e^{b}{ }_{\nu} g^{\mu \nu}=\eta^{a b},
$$

the Lorentz group arises naturally as the group of linear transformations of the base

$$
e^{a \prime}=\Lambda_{b}^{a} e^{b} \sim\left(\eta_{b}^{a}+\sigma_{b}^{a}\right) e^{b},
$$

$\left(\sigma_{b}^{a}\right.$ are the infinitesimal transformations) that preserves orthonormality.

$$
\Lambda_{c}^{a} \Lambda_{d}^{b} \eta^{c d}=\eta^{a b}, \Rightarrow \sigma^{(a}{ }_{c} \eta^{b) c}=\sigma^{(a b)}=0 .
$$

In ref. [43], ${ }^{11}$ Weyl proposed to define fermionic fields $\psi$ as fields transforming in the spinorial representation of the Lorentz group that acts in the tangent and cotangent space, that is

$$
\delta_{\sigma} \psi \equiv \frac{1}{2} \sigma^{a b} \Gamma_{s}\left(M_{a b}\right) \psi,
$$

\footnotetext{
${ }^{10}$ See, for instance ref. [41] for a proof of the first law in the context of 5-dimensional supergravity and the role that $\Phi$ plays in it.

${ }^{11}$ Translated in ref. [44].
} 
where $\Gamma_{r}\left(M_{a b}\right)$ stands for the matrices that represent the generators of the Lorentz group $\left\{M_{a b}\right\}$ in the representation $r$. As is well-known, the generators in the spinorial representation can be constructed taking antisymmetrized products of the gamma matrices $\gamma^{a}$, $\gamma^{a b} \equiv \gamma^{[a} \gamma^{b]}$

$$
\Gamma_{s}\left(M_{a b}\right)=\frac{1}{2} \gamma_{a b}, \quad \Rightarrow \quad \delta_{\sigma} \psi \equiv \frac{1}{4} \sigma^{a b} \gamma_{a b} \psi .
$$

Since these transformations can be different at each point, the Lorentz parameters $\sigma^{a b}$ take different values at different points of the spacetime and become functions $\sigma^{a b}(x)$ which will be smooth if the bases of the tangent and cotangent space are assumed to vary smoothly so that they are smooth vector and 1-form fields.

Theories containing fermionic fields in curved spacetimes are required to be invariant under these local Lorentz transformations. Their construction demands the introduction of a gauge field, the so-called spin connection 1-form, conventionally denoted by $\omega^{a b}=\omega_{\mu}^{a b} d x^{\mu}$. The spin connection enters the Lorentz-covariant derivatives of any field $T$ (indices not shown) transforming in the representation $r$ of the Lorentz group as follows:

$$
\mathcal{D} T^{(r)} \equiv\left[d-\frac{1}{2} \omega^{a b} \Gamma_{r}\left(M_{a b}\right)\right] T^{(r)}
$$

The transformation properties of $T^{(r)}$ are preserved by the covariant derivative if, under infinitesimal local Lorentz transformations,

$$
\delta_{\sigma} \omega^{a b}=\mathcal{D} \sigma^{a b}=\left[d-\frac{1}{2} \omega^{c d} \Gamma_{A d j}\left(M_{c d}\right)\right] \sigma^{a b}=d \sigma^{a b}-2 \omega^{[a}{ }_{c} \sigma^{|c| b]} .
$$

From now on $\nabla_{\mu}$ will denote the full (affine plus Lorentz) covariant derivative satisfying the first Vielbein postulate

$$
0=\nabla_{\mu} e^{a}{ }_{\nu} \equiv \partial_{\mu} e^{a}{ }_{\nu}-\omega_{\mu}^{a} b^{b} e_{\nu}-\Gamma_{\mu \nu}{ }^{\rho} e^{a}{ }_{\rho} .
$$

On pure Lorentz tensors $\nabla=\mathcal{D}$.

Now, how do spinors and general Lorentz tensors transform under infinitesimal g.c.t.s generated by an vector field $\xi$ ?

Customarily, these fields are treated as scalars, so that, if $£_{\xi}$ stands for the standard Lie derivative,

$$
\delta_{\xi} T=-£_{\xi} T=-\imath_{\xi} d T .
$$

There are many reasons why this has to be wrong. For starters, if we consider the particular case of a vector field $\xi$ generating a global Lorentz transformation in Minkowski spacetime $\xi^{\mu}=\sigma^{\mu}{ }_{\nu} x^{\nu}+a^{\mu}$, the transformation in eq. (2.18) is completely different from the transformation of a Lorentz tensor

$$
\delta_{\sigma} T=\frac{1}{2} \sigma^{a b} \Gamma_{r}\left(M_{a b}\right) T .
$$

However, it should reduce to this if the Fermionic fields introduced in curved spacetimes via Weyl's prescription have anything to do with the standard special-relativistic Fermionic fields. 
Furthermore, it is clear that the effect of the g.c.t. eq. (2.18) on $T$ depends on the gauge, or, equivalently, on the choice of tangent space basis. In other words the expression for $\delta_{\xi}$ in eq. (2.18) is not covariant under local Lorentz transformations.

Indeed, Lorentz tensors are not scalar nor tensor fields under g.c.t.s. They are sections of some bundle or, at a more pedestrian level, they are fields that, under g.c.t.s, transform as world tensors up to a local Lorentz transformation whose parameter depends on the field and on the generator of the g.c.t. $\sigma_{\xi}^{a b}$.

Then, instead of eq. (2.18) we must write

$$
\delta_{\xi} T=-£_{\xi} T+\delta_{\sigma_{\xi}} T,
$$

where $\sigma_{\xi}^{a b}$ makes $\delta_{\xi} T$ covariant under further local Lorentz transformations.

The parameter of the compensating local Lorentz transformation that renders $\delta_{\xi} T$ covariant turns out to be given by ${ }^{12}$

$$
\sigma_{\xi}^{a b}=\imath_{\xi} \omega^{a b}-\nabla^{[a} \xi^{b]},
$$

and it should be compared with the parameter of the compensating $\mathrm{U}(1)$ gauge transformation $\chi_{\xi}$ in eq. (2.7). By analogy we can define the Lorentz-algebra-valued momentum map

$$
P_{\xi}^{a b} \equiv \nabla^{[a} \xi^{b]} .
$$

We will see that this object satisfies a generalization of the equation that defines the momentum map in the Maxwell case eq. (2.5).

It is natural to define the Lorentz-covariant Lie derivative (or Lie-Lorentz derivative) of any tensor $T$ with Lorentz and world indices with respect to a vector field $\xi$ as (minus) this transformation: ${ }^{13}$

$$
\mathbb{L}_{\xi} T \equiv-\delta_{\xi} T=£_{\xi} T-\delta_{\sigma_{\xi}} T .
$$

The properties of the Lie-Lorentz derivative on spinors are reviewed in refs. [27, 38]. Here we are mainly interested in the Lie-Lorentz derivatives of the Vielbein and the spin connection, specially with respect to Killing vectors. According to the general definition, and after trivial manipulations, we find that the Lie-Lorentz derivative of the Vielbein is proportional to the Killing equation

$$
\mathbb{L}_{\xi} e^{a}{ }_{\mu}=\frac{1}{2}\left(\nabla_{\mu} \xi^{a}+\nabla^{a} \xi_{\mu}\right)=\frac{1}{2} e^{a \nu}\left(\nabla_{\mu} \xi_{\nu}+\nabla_{\nu} \xi_{\mu}\right)
$$

and, therefore, it vanishes when $\xi$ is a Killing vector field, independent of the basis chosen, as we should have expected.

\footnotetext{
${ }^{12}$ After ref. [22], this parameter is often written in the equivalent, but less transparent, form

$$
\sigma_{\xi}^{a b}=-£_{\xi} e^{[a}{ }_{\mu} e^{b] \mu} .
$$

${ }^{13}$ The Lie-Lorentz derivative was originally introduced for spinor fields in refs. [23-26] and its definition was later extended to more general Lorentz tensors $T$ transforming in an arbitrary representation $r$ [27].
} 
We will use this equivalent differential-form expression for the above equation:

$$
\mathbb{L}_{\xi} e^{a}=\mathcal{D} \xi^{a}+P_{\xi}{ }_{b}{ }^{b} e^{b} .
$$

Let us now consider the Lie-Lorentz derivative of the spin connection $\omega^{a b}$. Taking into account the inhomogeneous form of the compensating Lorentz transformation for the spin connection eq. (2.16) we get $^{14}$

$$
\mathbb{L}_{\xi} \omega^{a b}=£_{\xi} \omega^{a b}-\mathcal{D} \sigma_{\xi}^{a b},
$$

where $\sigma_{\xi}^{a b}$ is with the same parameter eq. (2.21). After some massaging, we can rewrite it in a much more suggestive form

$$
\mathbb{L}_{\xi} \omega^{a b}=\imath_{\xi} R^{a b}+\mathcal{D} P_{\xi}^{a b},
$$

where the Lorentz curvature 2-form $R^{a b} \equiv \frac{1}{2} R_{\mu \nu}^{a b} d x^{\mu} \wedge d x^{\nu}$ is defined as

$$
R^{a b}=d \omega^{a b}-\omega^{a}{ }_{c} \wedge \omega^{c b},
$$

and where we have replaced $\nabla^{[a} \xi^{b]}$ by $P_{\xi}^{a b}$, according to the definition of eq. (2.22).

The left-hand side of eq. (2.27) can be shown to vanish identically when $\xi$ is a Killing vector field, because of the identity

$$
\xi^{\nu} R_{\nu \mu}^{a b}+\nabla_{\mu}\left(\nabla^{[a} \xi^{b]}\right)=\nabla^{[a}\left(\nabla^{b]} \xi_{\mu}+\nabla_{\mu} \xi^{b]}\right) .
$$

As desired, for Killing vectors $k$ we have $\mathbb{L}_{k} e^{a}=0$ and $\mathbb{L}_{k} \omega^{a b}=0$ and both statements are Lorentz-invariant. ${ }^{15}$

For Killing vectors, eq. (2.29) can also be written in the form

$$
\imath_{k} R^{a b}=-\mathcal{D} P_{k}^{a b},
$$

which is the generalization of eq. (2.5) and justifies our definition of momentum map eq. (2.22) for Killing vectors. The main difference with the Lie-Maxwell case is that here we have an explicit expression for $P_{\xi}^{a b}$ for any $\xi$.

In the context of asymptotically-flat stationary black holes, it is known that, when evaluated on the bifurcation sphere $\mathcal{B H}$

$$
P_{k}^{a b}=\nabla^{[a} k^{b]} \stackrel{\mathcal{B H}}{=} \kappa n^{a b},
$$

where $\kappa$ is the surface gravity and $n^{a b}$ is the binormal, normalized to satisfy $n^{a b} n_{a b}=-2$. The constant ${ }^{16} \kappa$ is related to the Lorentz momentum map just as the electric potential on the horizon was shown to be related to the Maxwell momentum map in section 2.1. This parallelism between zeroth laws was observed in [29].

\footnotetext{
${ }^{14}$ The same expression can be found if one considers the variation of the Levi-Civita spin connection as a function of the variation of the Vielbein, given by (minus) the Lie-Lorentz derivative in eq. (2.24).

${ }^{15}$ Observe that $\mathbb{L}_{\xi} \omega^{a b}$ transforms as a Lorentz tensor even though $\omega^{a b}$ is not (it is a connection).

${ }^{16}$ See ref. [45] for a proof of the constancy of $\kappa$ over the horizon (the standard zeroth law of black hole mechanics [8]) that makes use of the Einstein equations and the dominant energy condition and ref. [46] for a proof that does not, relaying only on the assumption of geodesic completeness of the null generators of the event horizon.
} 


\section{The Einstein-Maxwell action and the RNT solutions}

In this section we present the $d$-dimensional Einstein theory and the $d$-dimensional ReissnerNordström-Tangherlini (RNT) solutions we are going to study, in order to fix the conventions. We will first give the action and equations of motion in the standard tensorial form, and will then rewrite them in the differential-language form that we will use in the following section.

\subsection{Action and equations of motion}

Setting $G_{N}^{(d)}=1$ for simplicity, and choosing as basic dynamical fields the Vielbein $e^{a}{ }_{\mu}$ and the Maxwell field $A_{\mu}$, the action of the Einstein-Maxwell theory in $d$ spacetime dimensions

$$
S\left[e^{a}{ }_{\mu}, A_{\mu}\right]=\frac{1}{16 \pi} \int d^{d} x e\left[R(\omega, e)-\frac{1}{4} F^{2}\right],
$$

where $e \equiv \operatorname{det}\left(e^{a}{ }_{\mu}\right), R(\omega, e)$ is the Ricci scalar, defined in terms of the Levi-Civita spin connection $\omega_{\mu}^{a b},^{17}$ that is

$$
R(\omega, e)=e_{a}^{\mu} e_{b}^{\nu} R_{\mu \nu}^{a b}(\omega),
$$

where $R_{\mu \nu}^{a b}(\omega)$ is the curvature 2-form of the Levi-Civita spin connection, defined in eq. (2.28). The Levi-Civita spin connection (metric compatible and torsion-free, that is $\left.\mathcal{D} e^{a}=0\right)$ is given by

$$
\omega_{a b c}=e_{a}{ }^{\mu} \omega_{\mu b a}=-\Omega_{a b c}+\Omega_{b c a}-\Omega_{c a b}, \quad \Omega_{a b c}=e_{a}{ }^{\mu} e_{b}{ }^{\nu} \partial_{[\mu \mid} e_{c \mid \nu]} .
$$

Finally, $F^{2}=F_{a b} F^{a b}, F_{a b}=e_{a}{ }^{\mu} e_{b}{ }^{\nu} F_{\mu \nu}$ and $F_{\mu \nu}$ is defined in eq. (2.3).

The equations of motion are

$$
\begin{aligned}
E_{a}{ }^{\mu} & \equiv \frac{\delta S}{\delta e^{a}{ }_{\mu}}=-\frac{e}{8 \pi}\left(G_{a}{ }^{\mu}-\frac{1}{2} T^{a}{ }_{\mu}\right), \\
E^{\mu} & \equiv \frac{\delta S}{\delta A_{\mu}}=\frac{1}{16 \pi} \partial_{\nu}\left(e F^{\nu \mu}\right)
\end{aligned}
$$

where

$$
T_{a}{ }^{\mu}=F_{a b} F^{\mu b}-\frac{1}{4} e_{a}^{\mu} F^{2},
$$

is the electromagnetic field's energy-momentum tensor.

In differential-form language, the action eq. (3.1) is usually written in this form

$$
S\left[e^{a}, A\right]=\frac{(-1)^{d-1}}{16 \pi} \int\left[\frac{1}{(d-2) !} R^{a_{1} a_{2}} \wedge e^{a_{3}} \wedge \cdots \wedge e^{a_{d}} \epsilon_{a_{1} \cdots a_{d}}-\frac{1}{2} F \wedge \star F\right] \equiv \int \mathbf{L},
$$

although it is more convenient to rewrite the first (Einstein-Hilbert) term as

$$
\frac{1}{(d-2) !} R^{a_{1} a_{2}} \wedge e^{a_{3}} \wedge \cdots \wedge e^{a_{d}} \epsilon_{a_{1} \cdots a_{d}}=\star\left(e^{a} \wedge e^{b}\right) \wedge R_{a b}
$$

\footnotetext{
${ }^{17}$ We are using the second-order formalism.
} 
The $(d-1)$-form equations of motion (which we write in boldface) are given by

$$
\begin{aligned}
\mathbf{E}_{a} & =\frac{1}{16 \pi}\left\{\imath_{a} \star\left(e^{c} \wedge e^{d}\right) \wedge R_{c d}+\frac{1}{2}\left(\imath_{a} F \wedge \star F-F \wedge \imath_{a} \star F\right)\right\}, \\
\mathbf{E} & =-\frac{1}{16 \pi} d \star F
\end{aligned}
$$

where $\imath_{c}$ stands for $i_{e_{c}}$, where $e_{c}=e_{c}{ }^{\mu} \partial_{\mu}$.

\subsection{The Reissner-Nordström-Tangherlini solutions}

The $d$-dimensional RNT solutions with rationalized mass $M$ and electric charge $q$ are described by the following metric and electromagnetic fields [19, 20, 47]:

$$
d s^{2}=\lambda d t^{2}-\frac{d r^{2}}{\lambda}-r^{2} d \Omega_{(d-2)}^{2}, \quad F_{t r}=\frac{16 \pi}{\omega_{(d-2)}} \frac{q}{r^{d-2}}
$$

where $d \Omega_{(d-2)}^{2}$ is the metric of the round $(d-2)$-sphere of unit radius, $\omega_{(d-2)}$ is its volume and

$$
\begin{aligned}
\lambda & =\frac{\left(r^{d-3}-r_{+}^{d-3}\right)\left(r^{d-3}-r_{-}^{d-3}\right)}{r^{2(d-3)}}, \\
r_{ \pm}^{d-3} & =\frac{8 \pi}{(d-2) \omega_{(d-2)}} M \pm r_{0}^{d-3}, \\
r_{0}^{d-3} & =\frac{8 \pi}{(d-2) \omega_{(d-2)}} \sqrt{M^{2}-\frac{2(d-2)}{(d-3)} q^{2}} .
\end{aligned}
$$

The origin of the annoying normalization factors lies in the standard normalization factor $(16 \pi)^{-1}$ of the action, which should be replaced by $\left[2(d-2) \omega_{(d-2)}\right]^{-1}$. Instead, we can just define

$$
\mathcal{M} \equiv \frac{8 \pi}{(d-2) \omega_{(d-2)}} M, \quad \mathcal{Q} \equiv \frac{16 \pi}{\omega_{(d-2)}} q,
$$

getting somewhat simpler expressions

$$
\begin{aligned}
F_{t r} & =\frac{\mathcal{Q}}{r^{d-2}}, \\
r_{ \pm}^{d-3} & =\mathcal{M} \pm r_{0}^{d-3} \\
r_{0}^{d-3} & =\sqrt{\mathcal{M}^{2}-\frac{\mathcal{Q}^{2}}{2(d-2)(d-3)}} .
\end{aligned}
$$

The event horizon of these solutions exists when $\mathcal{M} \geq[2(d-2)(d-3)]^{-1 / 2}|\mathcal{Q}|$ and then it is located at $r=r_{+}$and its surface gravity is given by

$$
\kappa=(d-3) r_{0}^{d-3} / r_{+}^{d-2} .
$$

The surface gravity vanishes in the extremal limit $r_{0}=0$, which is reached when $\mathcal{M}=$ $[2(d-2)(d-3)]^{-1 / 2}|\mathcal{Q}|$. We will always assume that $\kappa \neq 0$. 
The timelike Killing vector that becomes null on the horizon is $k=\partial_{t}$ in these coordinates, but they do not cover the bifurcate sphere because this expression for $k$ never vanishes. In the region covered by these coordinates we find that

$$
P_{k}{ }^{\mu \nu}=\nabla^{[\mu} k^{\nu]}=-\partial_{r} \lambda g^{\mu \nu}{ }_{r t} \stackrel{\mathcal{H}}{=} \kappa n^{\mu \nu},
$$

where the binormal takes the value

$$
n^{\mu \nu}=-2 g^{\mu \nu}{ }_{r t}, \quad \Rightarrow \quad n^{\mu \nu} n_{\mu \nu}=-2 .
$$

On the other hand, $\imath_{k} F=F_{t r} d r$ and

$$
P_{k}=\frac{\mathcal{Q} /(d-3)}{r^{d-3}} \stackrel{\mathcal{H}}{=} \frac{\mathcal{Q} /(d-3)}{r_{+}^{d-3}}=\Phi .
$$

In order to reach the bifurcation sphere we need to use Kruskal-Szekeres coordinates. For $d=4$ the change from $r, t$ to Kruskal-Szekeres's $U, V$ is known and given explicitly, for instance, in ref. [48]. To work in arbitrary $d$ we will just work near the event horizon: expanding the solution in eq. (3.9) around $r=r_{+}$and ignoring terms of second or higher order in $r-r_{+}$we get

$$
\begin{aligned}
d s^{2} & =2 \kappa\left(r-r_{+}\right) d t^{2}-\frac{d r^{2}}{2 \kappa\left(r-r_{+}\right)}-r_{+}^{2}\left[1+2\left(r-r_{+}\right) / r_{+}\right] d \Omega_{(d-2)}^{2}+\mathcal{O}\left(r-r_{+}\right)^{2}, \\
F_{t r} & =\frac{\mathcal{Q}}{r_{+}^{d-2}}\left[1-(d-2)\left(r-r_{+}\right) / r_{+}\right]+\mathcal{O}\left(r-r_{+}\right)^{2}
\end{aligned}
$$

The tortoise coordinate $r_{*}$ is

$$
r_{*}=\frac{1}{2 \kappa} \log \left(\frac{r-r_{+}}{r_{+}}\right)+C+\mathcal{O}\left(r-r_{+}\right)^{2},
$$

where $C$ is an integration constant that we set to zero for the sake of convenience. Defining

$$
v \equiv t+r_{*}, \quad u \equiv t-r_{*},
$$

the solution takes the form

$$
\begin{aligned}
& d s^{2}=2 \kappa r_{+} e^{\kappa(v-u)} d u d v-r_{+}^{2}\left[1+2 e^{\kappa(v-u)}\right] d \Omega_{(d-2)}^{2}+\mathcal{O}\left(r-r_{+}\right)^{2} \\
& F_{u v}=\kappa \frac{\mathcal{Q}}{r_{+}^{d-3}} e^{\kappa(v-u)}+\mathcal{O}\left(r-r_{+}\right)^{2} .
\end{aligned}
$$

Finally, we define the coordinates $U, V$

$$
V \equiv \sqrt{r_{+} / \kappa} e^{\kappa v}, \quad U \equiv-\sqrt{r_{+} / \kappa} e^{-\kappa u},
$$

in terms of which the solution takes the form

$$
\begin{aligned}
d s^{2} & =-2 d U d V-r_{+}^{2}\left[1-2 \kappa U V / r_{+}\right] d \Omega_{(d-2)}^{2}+\mathcal{O}(U V)^{2} \\
F_{U V} & =-\frac{\mathcal{Q}}{r_{+}^{d-2}}+\mathcal{O}(U V)^{2}
\end{aligned}
$$


The Killing vector $k=\partial_{t}$ becomes, in these coordinates

$$
k=\kappa\left(V \partial_{V}-U \partial_{U}\right)+\mathcal{O}(U V)^{2}, \quad \hat{k} \equiv k_{\mu} d x^{\mu}=\kappa(V d U-U d V)+\mathcal{O}(U V)^{2} .
$$

In these coordinates, the hypersurface $U=0$ is the past event horizon $\mathcal{H}^{-}$, generated by $\left.k\right|_{\mathcal{H}^{-}}=\kappa V \partial_{V}=\partial_{v}$. The hypersurface $V=0$ is the future event horizon $\mathcal{H}^{+}$generated by $\left.k\right|_{\mathcal{H}^{+}}=-\kappa U \partial_{U}=\partial_{u}$. They cross at the bifurcation sphere, which is defined by $U=V=0$ and can also be characterized as the spatial cross section of the horizon at which $k=0$.

On the other hand,

$$
\begin{aligned}
P_{k \mu \nu} d x^{\mu} \wedge d x^{\nu} & =d \hat{k}=2 \kappa d V \wedge d U+\mathcal{O}(U V)^{2}=2 \kappa g_{V U, \mu \nu} d x^{\mu} \wedge d x^{\nu}+\mathcal{O}(U V)^{2}, \\
\Rightarrow \quad n_{\mu \nu} & =-2 g_{U V, \mu \nu} .
\end{aligned}
$$

On the other hand,

$$
\begin{aligned}
\imath_{k} F & =\kappa \frac{\mathcal{Q}}{r_{+}^{d-2}}(V d U+U d V)+\mathcal{O}(U V)^{2}, \\
\Rightarrow \quad P_{k} & =C+\kappa \frac{\mathcal{Q}}{r_{+}^{d-2}} U V+\mathcal{O}(U V)^{2} .
\end{aligned}
$$

The constant $C$ clearly has to be identified with the electric potential over the horizon $\Phi$ in eq. (3.16). As observed in ref. [21], if we use the simplest choice of electromagnetic potential

$$
A=\frac{\mathcal{Q} /(d-3)}{r^{d-3}} d t
$$

we obtain,

$$
A=\frac{\mathcal{Q}}{2(d-3) \kappa r_{+}^{d-3}}\left[1+(d-3) \kappa U V / r_{+}+\mathcal{O}(U V)^{2}\right]\left(\frac{d V}{V}-\frac{d U}{U}\right),
$$

which is singular at the horizon.

\section{Wald-Noether charge for the E-M theory}

The general variation of the action of the Einstein-Maxwell theory eq. (3.6) is

$$
\delta S=\int\left\{\mathbf{E}_{a} \wedge \delta e^{a}+\mathbf{E} \wedge \delta A+d \mathbf{\Theta}(e, A, \delta e, \delta A)\right\},
$$

where $\mathbf{E}_{a}$ and $\mathbf{E}$ are, respectively, the $(d-1)$-form Einstein (3.8a) and Maxwell (3.4b) equations multiplied by the volume form $d^{d} x$ and

$$
\boldsymbol{\Theta}(e, A, \delta e, \delta A) \equiv-\frac{1}{16 \pi}\left[\star\left(e^{a} \wedge e^{b}\right) \wedge \delta \omega_{a b}-\star F \wedge \delta A\right],
$$

is the presymplectic $(d-1)$-form defined in ref. [10] and $\star$ stands for the Hodge dual. For the transformations given by (minus) the covariant Lie derivatives in eqs. (2.8), (2.25) and $(2.27)$

$$
\delta_{\xi} S=\int\left\{-\mathbf{E}_{a} \wedge\left(\mathcal{D} \xi^{a}+P_{\xi}^{a} b e^{b}\right)-\mathbf{E} \wedge\left(\imath_{\xi} F+d P_{\xi}\right)+d \mathbf{\Theta}\left(e, A, \delta_{\xi} e, \delta_{\xi} A\right)\right\},
$$


with

$$
\boldsymbol{\Theta}\left(e, A, \delta_{\xi} e, \delta_{\xi} A\right)=\frac{1}{16 \pi}\left[\star\left(e^{a} \wedge e^{b}\right) \wedge\left(\iota_{\xi} R^{a b}+\mathcal{D} P_{\xi}^{a b}\right)-\star F \wedge\left(\imath_{\xi} F+d P_{\xi}\right)\right] .
$$

Let us consider the first term. It is not difficult to see that $\mathbf{E}_{a} \wedge e^{b} P_{\xi}{ }^{a}{ }_{b}=0$ because the tensor contracted with the Lorentz momentum map give the Einstein equations, which are symmetric in the indices $a$ and $b$. The rest can be integrated by parts,

$$
-\mathbf{E}_{a} \wedge \mathcal{D} \xi^{a}=-(-1)^{d-1} d\left(\mathbf{E}_{a} \xi^{a}\right)+(-1)^{d-1} \xi^{a} \mathcal{D} \mathbf{E}_{a} .
$$

Using the Bianchi identity $\mathcal{D} R^{a b}=0$,

$$
\begin{aligned}
\xi^{a} \mathcal{D} \mathbf{E}_{a} & =\frac{1}{32 \pi} \xi^{a} \mathcal{D}\left(\imath_{a} F \wedge \star F-F \wedge \imath_{a} \star F\right) \\
& =\frac{1}{32 \pi} \xi^{a}\left[\nabla \imath_{a} F \wedge \star F-\imath_{a} F \wedge \nabla \star F-\nabla F \wedge \imath_{a} \star F-F \wedge \nabla \imath_{a} \star F\right],
\end{aligned}
$$

where we have replaced $\mathcal{D}$ by $\nabla$ is the exterior total covariant derivative operator which satisfies the first Vielbein postulate. Then, using the property

$$
\nabla \imath_{a} \omega=-\imath_{a} d \omega+\nabla_{a} \omega
$$

and replacing $\nabla$ by the exterior derivative when it acts on differential forms with no indices, as well as using the Bianchi identity $d F=0$, we get

$$
\xi^{a} \mathcal{D} \mathbf{E}_{a}=\frac{1}{32 \pi} \xi^{a}\left[\nabla_{a} F \wedge \star F-\imath_{a} F \wedge d \star F+F \wedge \imath_{a} d \star F-F \wedge \nabla_{a} \star F\right] .
$$

Since $\nabla_{a}$ commutes with the Hodge dual and $F \wedge \star G$ is symmetric in $F$ and $G$ for any 2 -forms $F, G$, the two terms with $\nabla_{a}$ cancel each other. Furthermore,

$$
F \wedge \imath_{a} d \star F=\imath_{a}(F \wedge d \star F)-\imath_{a} F \wedge d \star F
$$

and

$$
\xi^{a} \imath_{a} \omega=\imath_{\xi} \omega
$$

for any $p$-form, we arrive at

$$
(-1)^{d-1} \xi^{a} \mathcal{D} \mathbf{E}_{a}=-\frac{1}{16 \pi} d \star F \wedge \imath_{\xi} F .
$$

The second term in eq. (4.3) gives

$$
-\mathbf{E} \wedge\left(\imath_{\xi} F+d P_{\xi}\right)=\frac{1}{16 \pi} d \star F \wedge \imath_{\xi} F-(-1)^{d-1} d\left(\mathbf{E} P_{\xi}\right),
$$

and, collecting the partial results, we get

$$
\delta S_{\xi}=\int d \Theta^{\prime}\left(e, A, \delta_{\xi} e, \delta_{\xi} A\right)
$$


where

$$
\begin{aligned}
\boldsymbol{\Theta}^{\prime}\left(e, A, \delta_{\xi} e, \delta_{\xi} A\right) \equiv & \boldsymbol{\Theta}\left(e, A, \delta_{\xi} e, \delta_{\xi} A\right)+(-1)^{d}\left(\mathbf{E}_{a} \xi^{a}+\mathbf{E} P_{\xi}\right) \\
= & \frac{1}{16 \pi}\left[\star\left(e^{a} \wedge e^{b}\right) \wedge\left(\imath_{\xi} R_{a b}+\mathcal{D} P_{\xi a b}\right)-\star F \wedge\left(\imath_{\xi} F+d P_{\xi}\right)\right. \\
& +(-1)^{d} \imath_{\xi} \star\left(e^{a} \wedge e^{b}\right) \wedge R_{a b}+\frac{(-1)^{d}}{2}\left(\imath_{\xi} F \wedge \star F-F \wedge \imath_{\xi} \star F\right) \\
& \left.+(-1)^{d-1} d \star F P_{\xi}\right] \\
= & \left.-\imath_{\xi} \mathbf{L}+\frac{(-1)^{d-1}}{16 \pi} d \star \star F P_{\xi}-\star\left(e^{a} \wedge e^{b}\right) P_{\xi a b}\right] .
\end{aligned}
$$

The action of the Einstein-Maxwell theory eq. (3.6) is exactly invariant under local Lorentz and electromagnetic gauge transformations and it is invariant up to a total derivative under diffeomorphisms. Therefore, under the combined transformations $\delta_{\xi} \equiv-\mathbb{L}_{\xi}$ with the covariant Lie derivatives defined in eqs. (2.8), (2.25) and (2.27),

$$
\delta_{\xi} S=-\int d \imath_{\xi} \mathbf{L} .
$$

Taking into account the result in eq. (4.13), the arbitrariness of the domain of integration, of the parameter $\xi$, and the fact that we have not used the equations of motion, we conclude that, if we define the $(d-1)$-form

$$
\mathbf{J} \equiv \boldsymbol{\Theta}^{\prime}\left(e, A, \delta_{\xi} e, \delta_{\xi} A\right)+\imath_{\xi} \mathbf{L}
$$

it satisfies

$$
d \mathbf{J}=0,
$$

identically, off-shell. This, in its turn, implies the existence of a $(d-2)$-form $\mathbf{Q}[\xi]$ (the Wald-Noether charge) such that

$$
\mathbf{J}=d \mathbf{Q}[\xi] .
$$

The last line of eq. (4.14) gives the following expression for the Wald-Noether charge:

$$
\mathbf{Q}[\xi]=\frac{(-1)^{d-1}}{16 \pi}\left[\star F P_{\xi}-\star\left(e^{a} \wedge e^{b}\right) P_{\xi a b}\right] .
$$

\section{The first law of black hole mechanics in the E-M theory}

Following ref. [10] we define the pre-symplectic $(d-1)$-form

$$
\omega\left(\phi, \delta_{1} \phi, \delta_{2} \phi\right) \equiv \delta_{1} \boldsymbol{\Theta}\left(\phi, \delta_{2} \phi\right)-\delta_{2} \boldsymbol{\Theta}\left(\phi, \delta_{1} \phi\right),
$$

where $\phi$ stands for the Vielbein and Maxwell fields, and the symplectic form relative to the Cauchy surface $\Sigma$

$$
\Omega\left(\phi, \delta_{1} \phi, \delta_{2} \phi\right) \equiv \int_{\Sigma} \omega\left(\phi, \delta_{1} \phi, \delta_{2} \phi\right)
$$


Following now ref. [12], when $\phi$ solves the equations of motion $\mathbf{E}_{\phi}=0$, for any variation of the fields $\delta_{1} \phi=\delta \phi$ and the variations under diffeomorphisms $\delta_{2} \phi=\delta_{\xi} \phi$

$$
\omega\left(\phi, \delta \phi, \delta_{\xi} \phi\right)=\delta \mathbf{J}+d l_{\xi} \mathbf{\Theta}^{\prime}=\delta d \mathbf{Q}[\xi]+d l_{\xi} \boldsymbol{\Theta}^{\prime},
$$

where, in our case, $\mathbf{J}$ is given by eq. (4.18), $\boldsymbol{\Theta}^{\prime}$ is given in eq. (4.14) and we observe that, on-shell, $\boldsymbol{\Theta}=\boldsymbol{\Theta}^{\prime}$. Then, if $\delta \phi$ satisfies the linearized equations of motion, $\delta d \mathbf{Q}=d \delta \mathbf{Q}$. Furthermore, if the parameter $\xi=k$ generates a transformation that leaves invariant all the fields of the theory, $\delta_{k} \phi=0, \omega\left(\phi, \delta \phi, \delta_{k} \phi\right)=0$, and we arrive at

$$
d\left(\delta \mathbf{Q}[k]+\imath_{k} \mathbf{\Theta}^{\prime}\right)=0,
$$

which, when integrated over a hypersurface $\Sigma$ with boundary $\delta \Sigma$, gives

$$
\int_{\delta \Sigma}\left(\delta \mathbf{Q}[k]+\imath_{k} \mathbf{\Theta}^{\prime}\right)=0 .
$$

In our case, we are dealing with asymptotically flat, static black holes. $k$ is the timelike Killing vector whose Killing horizon coincides with the event horizon and the hypersurface $\Sigma$ is the space between infinity and the bifurcation sphere $(\mathcal{B H})$ on which $k=0$. Infinity and the bifurcate horizon are the two disconnected components of $\delta \Sigma$ and taking into account that $k=0$ on the bifurcation sphere, we obtain

$$
\delta \int_{\mathcal{B H}} \mathbf{Q}[k]=\int_{\infty}\left(\delta \mathbf{Q}[k]+\imath_{k} \mathbf{\Theta}^{\prime}\right) .
$$

As explained in ref. [12], the right-hand side can be identified with $\delta M$, where $M$ is the total mass of the black-hole spacetime. Using eq. (4.19), we find

$$
\delta \int_{\mathcal{B H}} \mathbf{Q}[k]=\frac{(-1)^{d-1}}{16 \pi} \delta \int_{\mathcal{B H}} \star F P_{k}+\frac{(-1)^{d}}{16 \pi} \delta \int_{\mathcal{B H}} \star\left(e^{a} \wedge e^{b}\right) P_{k a b} .
$$

According to the discussion at the end of section 2.1, $P_{k}$ can be identified with the electric potential $\Phi$ and it is constant over the horizon. The electric charge contained inside the horizon is given by

$$
\mathcal{Q} \equiv \frac{(-1)^{d-1}}{16 \pi} \int_{\mathcal{B H}} \star F,
$$

and the first term just gives $+\Phi \delta \mathcal{Q}$, which implies that we get a first-law-like relation if the second term gives $T \delta S$. Let us study that term. Using eq. (2.31) we get

$$
\begin{aligned}
\frac{(-1)^{d}}{16 \pi} \delta \int_{\mathcal{B H}} \star\left(e^{a} \wedge e^{b}\right) P_{k a b} & =\frac{(-1)^{d} \kappa}{16 \pi} \delta \int_{\mathcal{B H}} \star\left(e^{a} \wedge e^{b}\right) n_{a b} \\
& =-\frac{\kappa}{16 \pi} \delta \int_{\mathcal{B H}} d^{d-2} S n_{a b} n^{a b} \\
& =T \delta A / 4
\end{aligned}
$$

where we have used the normalization of the binormal $n_{a b} n^{a b}=-2, A$ is the area of the horizon and $T=\kappa / 2 \pi$ is the Hawking temperature.

Thus, we recover the first law of black hole mechanics if we identify the black hole entropy with one quarter of the area of the horizon. 


\section{Discussion}

In this paper we have shown how to define gauge-covariant Lie derivatives with the momentum map and how to use these derivatives in the proof of the first law of black-hole mechanics in the simple case of the Einstein-Maxwell theory with the Vielbein as the gravitational field. We have also shown that the momentum maps we have introduced in this case satisfy (well known) zeroth laws.

While the formulation of the first law of black-hole mechanics in the Einstein-Maxwell theory is certainly not new, our proposal for dealing with fields with gauge freedoms is a first step towards a generalization of the first law to more complex cases involving $p$ form fields with Chern-Simons terms such as those occurring in the Heterotic Superstring effective action. Work in this direction is in progress [34].

\section{Acknowledgments}

This work has been supported in part by the MCIU, AEI, FEDER (UE) grants PGC2018095205-B-I00 and PGC2018-096894-B-100, by the Spanish Research Agency (Agencia Estatal de Investigación) through the grant IFT Centro de Excelencia Severo Ochoa SEV2016-0597 and by the Principau D'Asturies grant FC-GRUPIN-IDI/2018/000174. The work of ZE has also received funding from "la Caixa" Foundation (ID 100010434), under the agreement LCF/BQ/DI18/11660042. TO wishes to thank M.M. Fernández for her permanent support.

Open Access. This article is distributed under the terms of the Creative Commons Attribution License (CC-BY 4.0), which permits any use, distribution and reproduction in any medium, provided the original author(s) and source are credited.

\section{References}

[1] A.C. Wall, A Survey of Black Hole Thermodynamics, arXiv:1804.10610 [INSPIRE].

[2] J.D. Bekenstein, Black holes and the second law, Lett. Nuovo Cim. 4 (1972) 737 [InSPIRE].

[3] J.D. Bekenstein, Black holes and entropy, Phys. Rev. D 7 (1973) 2333 [InSPIRE].

[4] D. Christodoulou, Reversible and irreversible transforations in black hole physics, Phys. Rev. Lett. 25 (1970) 1596 [INSPIRE].

[5] D. Christodoulou and R. Ruffini, Reversible transformations of a charged black hole, Phys. Rev. D 4 (1971) 3552 [INSPIRE].

[6] S.W. Hawking, Gravitational radiation from colliding black holes, Phys. Rev. Lett. 26 (1971) 1344 [INSPIRE].

[7] S.W. Hawking, Black holes in general relativity, Commun. Math. Phys. 25 (1972) 152 [INSPIRE].

[8] J.M. Bardeen, B. Carter and S.W. Hawking, The Four laws of black hole mechanics, Commun. Math. Phys. 31 (1973) 161 [InSPIRE]. 
[9] S.W. Hawking, Particle Creation by Black Holes, Commun. Math. Phys. 43 (1975) 199 [Erratum ibid. 46 (1976) 206] [INSPIRE].

[10] J. Lee and R.M. Wald, Local symmetries and constraints, J. Math. Phys. 31 (1990) 725 [INSPIRE].

[11] R.M. Wald, Black hole entropy is the Noether charge, Phys. Rev. D 48 (1993) 3427 [gr-qc/9307038] [INSPIRE].

[12] V. Iyer and R.M. Wald, Some properties of Noether charge and a proposal for dynamical black hole entropy, Phys. Rev. D 50 (1994) 846 [gr-qc/9403028] [INSPIRE].

[13] P.A. Cano, P. Meessen, T. Ortín and P.F. Ramírez, $\alpha^{\prime}$-corrected black holes in String Theory, JHEP 05 (2018) 110 [arXiv: 1803.01919] [INSPIRE].

[14] P.A. Cano, S. Chimento, P. Meessen, T. Ortín, P.F. Ramírez and A. Ruipérez, Beyond the near-horizon limit: Stringy corrections to Heterotic Black Holes, JHEP 02 (2019) 192 [arXiv: 1808.03651] [INSPIRE].

[15] J.D. Edelstein, K. Sfetsos, J. Sierra-Garcia and A. Vilar López, T-duality equivalences beyond string theory, JHEP 05 (2019) 082 [arXiv:1903.05554] [INSPIRE].

[16] P.A. Cano, S. Chimento, R. Linares, T. Ortín and P.F. Ramírez, $\alpha^{\prime}$ corrections of Reissner-Nordström black holes, JHEP 02 (2020) 031 [arXiv:1910.14324] [INSPIRE].

[17] Z. Elgood and T. Ortín, T duality and Wald entropy formula in the Heterotic Superstring effective action at first order in $\alpha^{\prime}$, arXiv: 2005.11272 [INSPIRE].

[18] T. Ortín, $O(n, n)$ invariance and Wald entropy formula in the Heterotic Superstring effective action at first order in $\alpha^{\prime}$, arXiv:2005.14618 [INSPIRE].

[19] H. Reissner, Über die Eigengravitation des elektrischen Feldes nach der Einsteinschen Theorie, Annals Phys. 50 (1916) 106.

[20] G. Nordström, On the Energy of the Gravitation field in Einstein's Theory, Proc. Kon. Ned. Akad. Wet. 20 (1918) 1238.

[21] S. Gao, The First law of black hole mechanics in Einstein-Maxwell and Einstein-Yang-Mills theories, Phys. Rev. D 68 (2003) 044016 [gr-qc/0304094] [INSPIRE].

[22] T. Jacobson and A. Mohd, Black hole entropy and Lorentz-diffeomorphism Noether charge, Phys. Rev. D 92 (2015) 124010 [arXiv:1507.01054] [INSPIRE].

[23] A. Lichnerowicz, Spineurs harmoniques, C. R. Acad. Sci. Paris 257 (1963) 7.

[24] Y. Kosmann, Dérivées de Lie des spineurs, C. R. Acad. Sci. Paris Sér. A 262 (1966) A289.

[25] Y. Kosmann, Dérivées de Lie des spineurs, Annali Mat. Pura Appl. (IV) 91 (1972) 317.

[26] D.J. Hurley and M.A. Vandyck, On the concepts of Lie and covariant derivatives of spinors. Part 1, J. Phys. A 27 (1994) 4569 [INSPIRE].

[27] T. Ortín, A Note on Lie-Lorentz derivatives, Class. Quant. Grav. 19 (2002) L143 [hep-th/0206159] [INSPIRE].

[28] P.B. Aneesh, S. Chakraborty, S.J. Hoque and A. Virmani, First law of black hole mechanics with fermions, arXiv: 2004.10215 [INSPIRE].

[29] K. Prabhu, The First Law of Black Hole Mechanics for Fields with Internal Gauge Freedom, Class. Quant. Grav. 34 (2017) 035011 [arXiv:1511.00388] [INSPIRE]. 
[30] K. Hajian and M.M. Sheikh-Jabbari, Solution Phase Space and Conserved Charges: A General Formulation for Charges Associated with Exact Symmetries, Phys. Rev. D 93 (2016) 044074 [arXiv: 1512.05584] [INSPIRE].

[31] G. Compere, Note on the First Law with p-form potentials, Phys. Rev. D 75 (2007) 124020 [hep-th/0703004] [INSPIRE].

[32] E. Frodden and D. Hidalgo, Surface Charges for Gravity and Electromagnetism in the First Order Formalism, Class. Quant. Grav. 35 (2018) 035002 [arXiv:1703.10120] [INSPIRE].

[33] E. Frodden and D. Hidalgo, Surface Charges Toolkit for Gravity, Int. J. Mod. Phys. D 29 (2020) 2050040 [arXiv: 1911.07264] [inSPIRE].

[34] Z. Elgood, P. Meessen and T.Ortín, The Wald entropy formula in the Heterotic Superstring Effective action at first order in $\alpha^{\prime}$, in preparation.

[35] M.A.J. Vandyck, On the problem of space-time symmetries in the theory of supergravity, Gen. Rel. Grav. 20 (1988) 261 [inSPIRE].

[36] M.A. Vandyck, On the problem of space-time symmetries in the theory of supergravity. 2: $N=2$ supergravity and spinorial Lie derivatives, Gen. Rel. Grav. 20 (1988) 905 [INSPIRE].

[37] J.M. Figueroa-O'Farrill, On the supersymmetries of Anti-de Sitter vacua, Class. Quant. Grav. 16 (1999) 2043 [hep-th/9902066] [INSPIRE].

[38] T. Ortín, Gravity and Strings, 2nd edition, Cambridge University Press (2015).

[39] I.A. Bandos and T. Ortín, On the dualization of scalars into $(d-2)$-forms in supergravity. Momentum maps, R-symmetry and gauged supergravity, JHEP 08 (2016) 135 [arXiv: 1605.05559] [INSPIRE].

[40] T.T. Wu and C.N. Yang, Concept of Nonintegrable Phase Factors and Global Formulation of Gauge Fields, Phys. Rev. D 12 (1975) 3845 [inSPIRE].

[41] H.K. Kunduri and J. Lucietti, The first law of soliton and black hole mechanics in five dimensions, Class. Quant. Grav. 31 (2014) 032001 [arXiv:1310.4810] [InSPIRE].

[42] V. Frolov and I. Novikov, eds., Black hole physics: Basic concepts and new developments, vol. 96 (1998), 10.1007/978-94-011-5139-9 [INSPIRE].

[43] H. Weyl, Electron and Gravitation, Z. Phys. 330 (1929) 56.

[44] L. O'Raifeartaigh, The Dawning of Gauge Theory, Princeton University Press, Princeton, New Jersey (1997).

[45] R.M. Wald, General Relativity, The University of Chigago Press (1992) [DOI].

[46] R.M. Wald, Black holes and thermodynamics, in Black Hole Physics, Proceedings of the NATO Advanced Study Institute on Black Hole Physics (12th Course of the International School of Cosmology and Gravitation ofthe Ettore Majorana Centre for Scientific Culture) Erice, Italy (1991), V. De Sabbata and Z. Zhang Eds., Kluwer Academic Publishers (1992).

[47] F.R. Tangherlini, Schwarzschild field in $n$ dimensions and the dimensionality of space problem, Nuovo Cim. 27 (1963) 636 [INSPIRE].

[48] S.W. Hawking and G.F.R. Ellis, The Large Scale Structure of Space-Time, Cambridge Monographs on Mathematical Physics, Cambridge University Press (1973) [DOI] [INSPIRE]. 